\title{
Research on Simulation Methods of Electric Field Intensity on Surface of 10 kV Cable Joint
}

\author{
Ruxin Zhang1, Jun Xiong1, Zheng Wu'1, Lei Liao', Mingyan Wu'1, Gang Du¹, Xueyou Huang1, \\ Wenpei Jin', Haiming Li² ${ }^{2}$ Jian Zhang ${ }^{2}$, Wenli Cheng², Binxian $\mathrm{Lu}^{2 *}$ \\ ${ }^{1}$ Guagnzhou Power Supply CSG, Guangzhou, China \\ ${ }^{2}$ Department of Electrical and Electronic Engineering, North China Electric Power University, Beijing, China \\ Email: vanishangel@163.com,404127726@qq.com,469298598@qq.com,33859515@qq.com,254689291@qq.com, \\ dudu-2566@163.com,hxy_you@sina.com,991740191@qq.com,lhm0210@ncpeu.edu.cn, zja@ncepu.edu.cn, \\ ccwl@ncepu.edu.cn, *lbx@ncepu.edu.cn
}

How to cite this paper: Zhang, R.X., Xiong, J., Wu, Z., Liao, L., Wu, M.Y., Du, G., Huang, X.Y., Jin, W.P., Li, H.M., Zhang, J., Cheng, W.L and Lu, B.X. (2020) Research on Simulation Methods of Electric Field Intensity on Surface of $10 \mathrm{kV}$ Cable Joint. Energy and Power Engineering, 12, 37-45.

https://doi.org/10.4236/epe.2020.124B004

Received: January 6, 2020

Accepted: April 7, 2020

Published: April 10, 2020

\begin{abstract}
The electric field intensity (EFI) is important characteristic quantity for evaluating the internal insulation state of cable joints. Based on finite element method, this paper proposes two EFI research methods, field-circuit coupling method and equivalent circuit method. The average EFI of the inner surface of the outer semi-conducting shield can be calculated from the current in the measuring circuit. The relative error between these two methods is about $15 \%$, which roughly proves the consistency of the two methods. Further practical application research enables online monitoring of cable joints.
\end{abstract}

\section{Keywords}

Cable Joints, Electric Field Intensity, Equivalent Circuit Method, Field-Circuit Coupling Method, Finite Element Simulation Software

\section{Introduction}

In recent years, with the development of urban modernization, the unit capacity has been increasing, and the share of power cables in urban power supply has increased [1]. In some urban areas, overhead transmission lines have been gradually replaced by laying cables. With the increase in the number of cables, a large number of cable accidents have been caused due to external force damage, insulation dampness, long-term overload operation, cable joint failure, environment and temperature, and the aging or natural disaster of the cable body [2]. Power outages in some power grids have caused a lot of inconvenience to factory production and people's lives, causing significant economic losses. Operating expe- 
rience data shows that joint failures account for $70 \%$ of the total number of failures, and its reliability directly affects the operating safety of power systems. Cable accessories include cable terminations and intermediate joints [3]. Due to the complexity of the structure, fabrication, connection, and operating conditions of the cable joint, it is easy to produce EFI concentration, which is a weak link for safe operation of power systems [4].

The electric field intensity (EFI) is important characteristic quantity for evaluating the internal insulation state of cable joints. When there is a defect in the insulation of the cable joint, the internal EFI distribution will change, and when the internal EFI exceeds the breakdown EFI, it will lead to the occurrence of partial discharge, and then accelerate the dielectric breakdown [5]. Therefore, it is of great significance to study the factors affecting the distribution of the EFI at the end of the cable for the safe operation of power cables, ensuring the reliability of power supply, reducing environmental pollution, and reducing manufacturing and operating costs. For many years, relevant scholars at home and abroad have been exploring the factors affecting the reliability of cables to improve the reliability of cable joints. However, because the joint structure and manufacturing process are complicated, the manufacturing cost is high, and there are problems of time consuming and laborious. Therefore, the simulation analysis of cable accessories is widely used [6]. The EFI research method proposed in this paper is based on finite element simulation software.

\section{Measuring Principle of EFI}

The source of the alternating electric field generated by power equipment is the charge on the metal surface of the power equipment. Due to the low frequency in the case of power frequency, with the exception of inductors and transformers, the effect of magnetic field changes with time in most power equipment can be ignored. In this case, the power frequency electric field is a quasi-static electric field, and the induced electric field is much smaller than the coulomb electric field. The field equation is

$$
\begin{gathered}
\nabla \times \vec{E}=0 . \\
\nabla \bullet \vec{D}=\rho . \\
\nabla \bullet(\vec{J}+\partial \vec{D} / \partial t)=0 .
\end{gathered}
$$

Auxiliary equations are

$$
\begin{aligned}
& \vec{J}=\gamma \vec{E} . \\
& \vec{D}=\varepsilon \vec{E} .
\end{aligned}
$$

where $\vec{E}$ is EFI vector, $\vec{J}$ is conductive current density vector, $\vec{D}$ is electric displacement vector, $\rho$ is volume charge density, $\varepsilon$ is the permittivity, $\gamma$ is conductivity, $t$ is time, $\nabla$ is Nabla operator.

In the case of power frequency, the wavelength is $6000 \mathrm{~km}$, which is much larger than the size of the conductor surface. Therefore, the phase difference 
between the electric field intensities between the points on the surface of the metal conductor can be ignored. If we are concerned about the EFI at point A on the surface of the conductor shown in Figure 1, we can perform a cutting process around this point, sand the edges, and then restore it to its original position completely with insulating glue. Ensure that this sheet is insulated from the body portion of the outer semi-conductive shield. In order to measure the conduction current, we use a resistor to connect this sheet with the other part of the conductor. This resistor is called measurement resistor.

As shown in Figure 2, $R$ is the resistance of measurement resistor, $u_{1}$ is the voltage across measurement resistor, $i_{\mathrm{R}}$ is the current flowing through the measuring resistor, $i_{\mathrm{c}_{1}}$ is the current across capacitance $C_{1}$, capacitance $C_{1}$ is the equivalent one between the slice and the outer conductor shield, capacitance $C_{2}$ is the equivalent one between the high voltage conductor and the slice, $u_{\mathrm{C}_{2}}$ is voltage across capacitance $C_{2}, i_{\mathrm{c}_{2}}$ is the total current.

In the case of sinusoidal steady state, according to relationship of voltage and current of capacitance, we can calculate the average EFI of the inner surface of the slice from Equation (6) [7].

$$
E=I /(\omega \cdot \varepsilon \cdot S) .
$$

Among them, $E$ is EFI on the inner surface of slice $(\mathrm{V} / \mathrm{m}), \varepsilon$ for the permittivity $(\mathrm{F} / \mathrm{m}), I$ is the total current (A) of the equivalent circuit in Figure 2, $S$ is the surface area of the slice $\left(\mathrm{m}^{2}\right), \omega$ is angular frequency $(\mathrm{rad} / \mathrm{s})$.

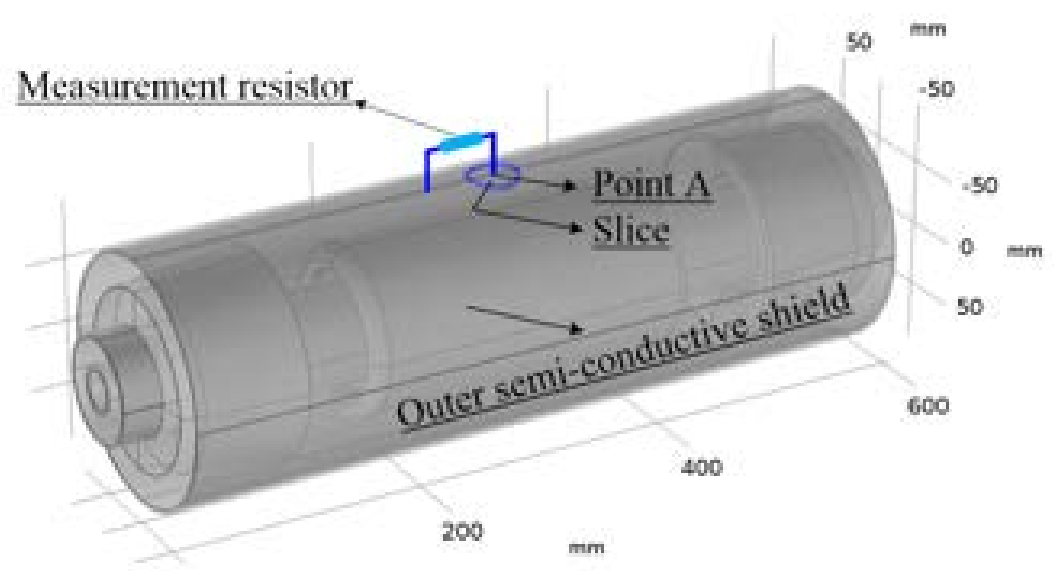

Figure 1. Schematic diagram of the measuring principle.
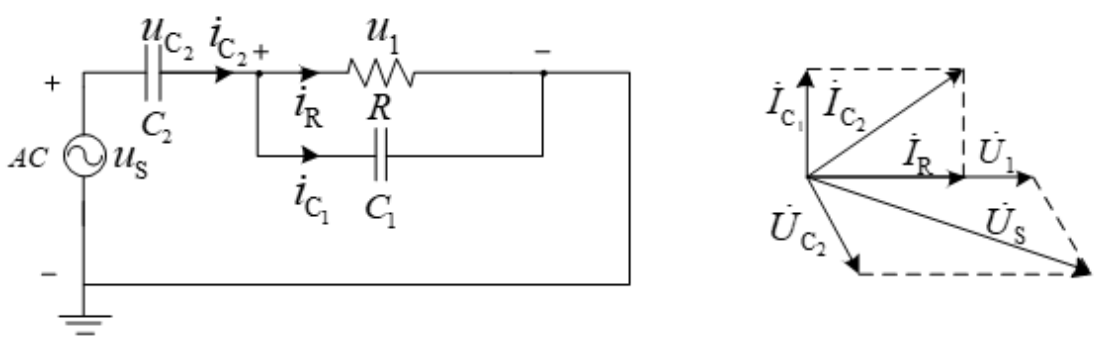

Figure 2. Equivalent circuit and phasor diagram of measurement principle. 


\section{Analysis Model}

The simulation model used in this paper is a three-dimensional $10 \mathrm{kV}$ cable joint as shown in Figure 5, and in order to clearly show the structural composition of the model and calculate the boundary conditions, the axisymmetric model of the cable joint in Figure 3 is used to briefly explain. The model is calculated using the finite element method, which mainly includes three parts: model pre-processing, model calculation and model post-processing. The pre-processing mainly includes the establishment of the geometric model and the meshing of the geometric model. The meshing of the geometric model is the key step to determine the calculation accuracy of the model.

\subsection{Establishment of Simulation Model and Mesh Division of $10 \mathrm{kV}$ Cable Joint}

As shown in Figure 3, the model consists of 9 parts, including: copper conductor, inner semi-conductive layer, main insulation, outer semi-conductive layer, stress cone, high-voltage shielding layer, reinforced insulation layer, and insulation shielding layer. The boundary conditions imposed by the model during calculation are shown in Figure 4. The material parameters of each component are shown in Table 1.

In addition, the model in this paper found that the meshing accuracy of the mesh near the slice has a greater impact on the calculation results, but the size of the slice is smaller than the entire model, so it is suitable to use local refinement. The method is divided to avoid the increase of calculation time caused by the overall mesh refinement and the limitation of memory and so on. Therefore, as shown in Figure 6, the slice and its surrounding area are inside a sphere in preparation for subsequent segmentation. This paper adopts the method of

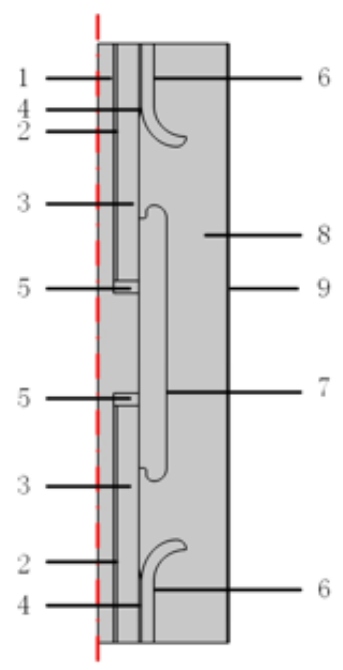

1. Copper conductor, 2. Inner semi-conductive layer, 3. Main insulation, 4. Outer semi-conductive layer, 5. Air inside of the joint, 6. Stress cone, 7. High voltage shield, 8. Reinforced insulation, 9. Outer semi-conductive shield

Figure 3. Schematic diagram of the $10 \mathrm{kV}$ cable joint. 


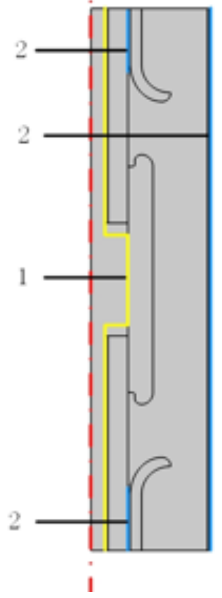

1. Conductor with high voltage, 2. Ground tab.

Figure 4. Boundary condition of the $10 \mathrm{kV}$ cable joint.

Table 1. Material parameter.

\begin{tabular}{cccc}
\hline \multirow{2}{*}{ Materials } & \multicolumn{3}{c}{ Parameter and application } \\
\cline { 2 - 4 } & Relative permittivity & Conductivity $(\mathrm{S} / \mathrm{m})$ & Application \\
\hline Copper & 1 & $5.8 \times 10^{8}$ & Cable conductor \\
XLPE & 2.3 & $1 \times 10^{-15}$ & Main insulation \\
Silicone Rubber & 4.3 & $2.727 \times 10^{-12} \times \mathrm{e}^{9.796 \times 10^{-6} \times \mathrm{E}}$ & Joint insulation \\
$\begin{array}{c}\text { Semi-conducting } \\
\text { material }\end{array}$ & 20 & 10 & $\begin{array}{c}\text { Conductive layers } \\
\text { and stress cone }\end{array}$ \\
Insulation glue & 3.5 & $1 \times 10^{-12}$ & Insulation gap \\
\hline
\end{tabular}

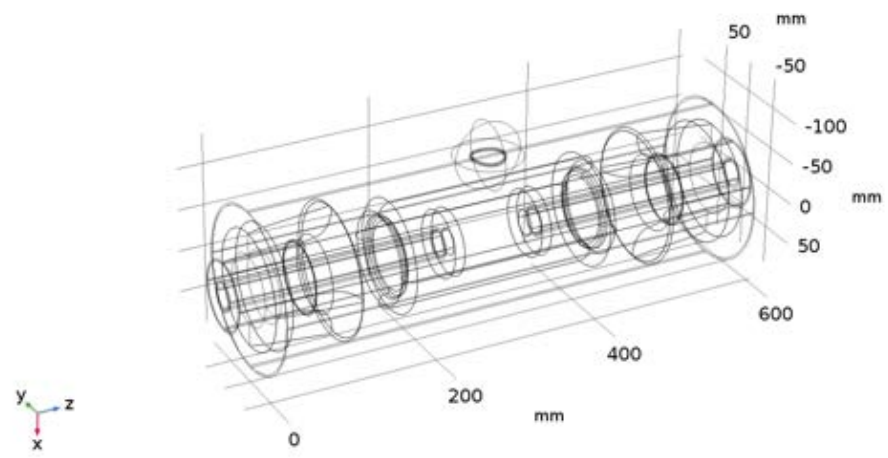

Figure 5. Simulation model of the $10 \mathrm{kV}$ cable joint.

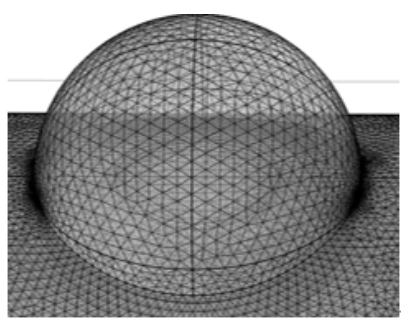

Figure 6. Local grid. 
applying multi-layer boundaries to the slice attachment area to refine the local grid and ensure the accuracy of the calculation without adding too much calculation time and computing memory.

\subsection{Equivalent Circuit and Field-Circuit Coupling Analysis Method}

When simulating the EFI of the inner surface of the outer semi-conducting shield of the $10 \mathrm{kV}$ cable joint, two simulation calculation methods can be used to solve it.

One method is use the electrostatic field module of the finite element simulation software to calculate the capacitance between each part of Figure 1 and then obtain the measurement circuit of Figure 2. The capacitance matrix of an electrical system allows us to evaluate cross talk between excitation ports. In this simulation calculation, we see copper conductor as terminal 1, slice as terminal 2, outer semi-conductive shield as terminal 3, which consist of a three-terminal system, we can excite one terminal and set the other two to ground. If we repeat this method of exciting one terminal at a time, since there are three terminals, we can evaluate nine possible values of capacitance. The capacitance component $C_{11}$ is the capacitance evaluated between the grounded terminals and Terminal 1. This can be calculated by exciting Terminal 1 . The capacitance between Terminals 1 and 2 would be $\mathrm{C}_{21}$. This can be calculated once we have information about $C_{11}$ and $C_{22}$. This means we would need to solve the model once again by exciting Terminal 2. By definition, $C_{21}$ and $C_{12}$ would be equal. This means that a three-terminal system will have six unique values of capacitance.

Through calculation, $C_{12}\left(C_{1}\right.$ in Figure 2) and $C_{23}\left(C_{2}\right.$ in Figure 2) can be obtained. By using capacitance $C_{1}, C_{2}$ and $R$, the measurement circuit can be formed.

The other method is to use the current field and circuit modules in the finite element simulation software, that is, the field-circuit coupling method to calculate the current through the measurement resistor, and then use Equation (6) to calculate the EFI of the inner surface of the slice corresponding to different power frequency voltage. It differs from the method described in part " 4.1 " in that the copper conductor is first processed with a power frequency voltage in current field, the shield is grounded, and the slice is connected to the circuit filed. In the circuit module, the slice voltage is set to the external I terminal 1 , and a $1 \mathrm{M} \Omega$ measurement resistor is connected between the external I terminal 1 and the ground. Referring to the value of capacitor $C_{1}$ in part " 4.1 " and comparing with resistor $R$, it can be calculated that almost all the current through the measuring circuit passes through resistor $R$. Therefore, in the case of applying a power frequency voltage to the copper conductor, it can be considered that the total current in the circuit is equal to the current on the measurement resistance, and the EFI of the inner surface of the slice is obtained using Equation (6).

\subsection{Calculation Steps}

The simulation calculations in this paper are mainly aimed at the EFI on the in- 
ner surface of the outer semi-conductive layer of the cable connector, and two different methods of field-circuit coupling and equivalent circuit are adopted to solve it.

Firstly, the simulation model is established, because the setting of the slice uses a three-dimensional model; in addition, the material parameters are set according to the physical properties of the $10 \mathrm{kV}$ cable joint; then the boundary conditions and loads are applied, and the boundary conditions are selected from the effective voltage value as the calculated load. The final simulation results are obtained through the analysis and calculation of the proposed method. The results obtained by different methods are compared and analyzed.

\section{Simulation Results and Analysis}

Using the first equivalent circuit method described in part "3.2", the capacitance matrix in the quasi-static electric field is solved to obtain the gap capacitance $C_{1}$ in the established simulation model and the capacitance $C_{2}$ between the semi-conducting layer outside the section, so as to form the measuring circuit with the measuring resistance $R$ and obtain the total current in the circuit. In addition, for the field-circuit coupling method, the simulation model is first studied in the case of power frequency, and then "current through device $R$ " can be obtained by global calculation of derived values. Since the current through the resistance $R$ in the circuit is nearly equal to the total current in the circuit, the results calculated by the two methods are compared using the "current through the device $R^{\prime \prime}$ in the field-circuit coupling and total current in equivalent circuit. With the change of power frequency voltage applied to copper conductor, the total current results calculated by the two methods are shown in Figure 7.

Using Equation (6), the average EFI of the inner surface of the outer semi-conducting shield can be calculated from the current in the measuring circuit. The comparison of the EFI calculated by the field-circuit coupling method

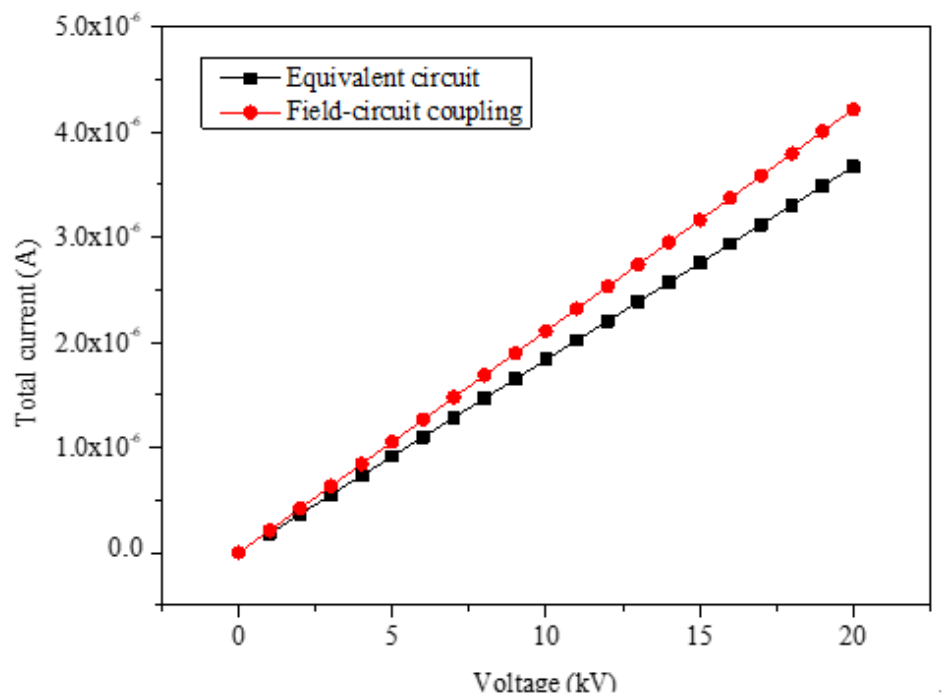

Figure 7. Total current contrast of measuring circuit under different voltage. 


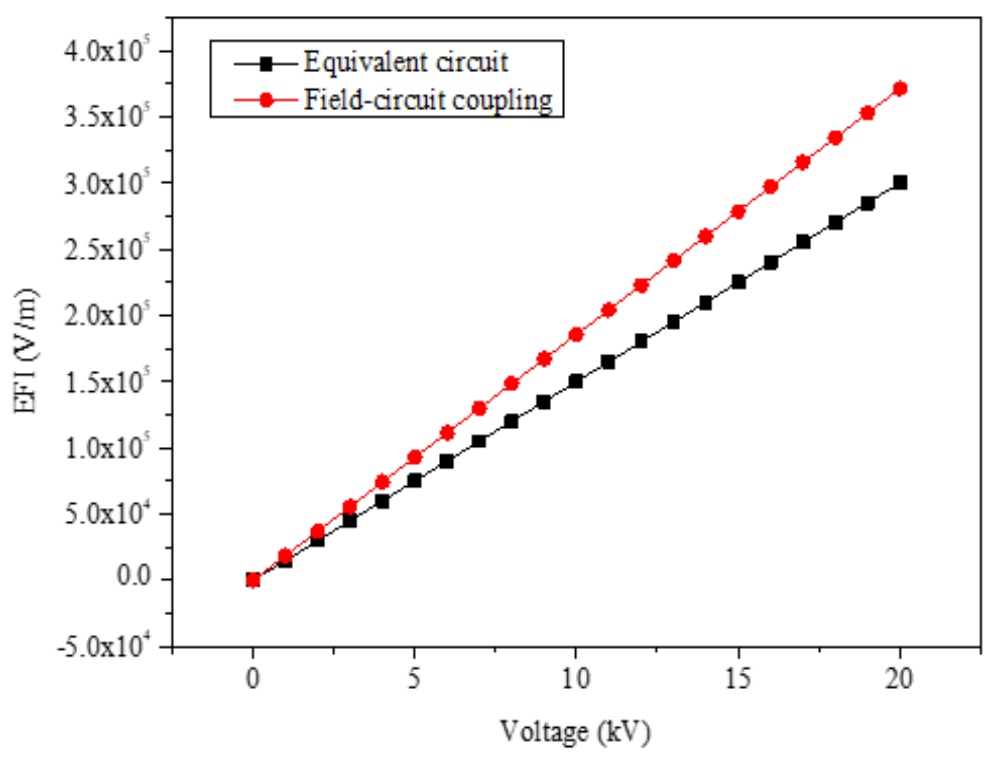

Figure 8. EFI contrast of inner surface of outer semi-conductive shield under different voltage.

and the equivalent circuit method is shown in Figure 8. The relative error between the two methods is about $15 \%$, which approximately proves the consistency of the two methods. In the following research, if we can find more methods to calculate the EFI based on the experimental results, and compare their results with the above two methods, the correctness of the calculation method in this paper will be more fully verified.

\section{Conclusions}

- In the case of power frequency, the relative error between the field-circuit coupling method and the equivalent circuit method is about $15 \%$, which approximately proves the consistency of the two methods. Although the results are not sufficient to prove the correctness of the calculation method, if we can find more methods to calculate the EFI based on the experimental results, the correctness of the calculation method in this paper will be more fully verified.

- For the actual $10 \mathrm{kV}$ cable joint, the structure proposed in this article can be used to modify its structure, and the voltage drop information on the measured resistor can be transmitted to the "command center" through the transmission line. In this way, the measurement signal is obtained, and the change of the EFI is used to realize the measurement and monitoring of the EFI of the cable joint. Further practical application research enables online monitoring of cable joints.

\section{Acknowledgements}

This work was supported by the Science and Technology Project of China South Power Gird Co., Ltd. (GZHKJXM20180140). 


\section{Conflicts of Interest}

The authors declare no conflicts of interest regarding the publication of this paper.

\section{References}

[1] Batalović, M., Zildžo, H., Matoruga, H., et al. (2019) Detection of Defect Presence inside the Insulation of Cable Accessories through Changes in Cable Capacitance. Proceedings of the 27 th International Conference on Information, Communication and Automation Technologies, Sarajevo, 1-4 October 2019, 2379-3791.

[2] Hwang, J., et al. (2016) Insulation Design of a Stop Joint Box of 80-kV DC HTS Cables Based on DC Electric Field Analysis. IEEE Transactions on Applied Superconductivity, 26, 1-12. http://ieeexplore.iee.org/10.1109/2015.2507122

[3] Das, S., Majumder, R. and Singh, S. (2017) Cable Joint Installation Time Optimization. IEEE Transactions on Dielectrics and Electrical Insulation, 24, 3959-3965. 1-12. http://ieeexplore.ieee.org/10.1109/2017.006776

[4] Gustavsen, B., Høyer-Hansen, M., Hatlo, M., et al. (2019) Voltages and AC Corrosion on Metallic Tubes in Umbilical Cables Caused by Magnetic Induction From Power Cable Charging Currents. IEEE Transactions on Power Delivery, 34, 596-605. http://ieeexplore.ieee.org/10.1109/2018.2881221

[5] Baek, S., Kim, H., Cho, J., et al. (2018) Cryogenic Electrical Insulation Characteristics of Solid Insulator for the HVDC HTS Cable. IEEE Transactions on Applied Superconductivity, 28, 2378-7074. http://ieeexplore.ieee.org/10.1109/2018.2824337

[6] Kim, W., Kim, H., Cho, J., et al. (2015) Electrical and Mechanical Characteristics of Insulating Materials for HTS DC Cable and Cable Joint. IEEE Transactions on Applied Superconductivity, 25, 1-4. http://ieeexplore.ieee.org/10.1109/2014.2385958

[7] Lu, B., Cheng, W., Xiong, J., et al. (2019) Research on Measurement Method of Electric Field Intensity on Surface of Metal Conductor. IEEE Transactions on Industrial Electronics, Early Access. http://ieeexplore.ieee.org/10.1109/2019.2938489 